\title{
Sulla propagazione di onde piane in magneto-termo-elasticità.
}

\author{
Nota di Sergio Levoni (a Modena) (*)
}

Sunto. - Si ricavano dapprima le equazioni della magneto-termo-elasticitò facendo uso del teorema delle quantità di moto per ottenere l'equazione delle piccole oscillazioni elastiche e del principio di conservazione dell'energia per giungere all' equazione generaliazata del calore. Dopo la linearizzazione delle equazioni in questione, si affronta il problema della propagazione di onde piane (supponendo cioè che tutte le grandezze abbiano la stessa dipendenza spazio-temporale di tipo sinoidale) ricorrendo a un procedimento che permette di ridursi a due sole equazioni, una vettoriale e una scalare, nella velocità, temperatura e densità della corrente elettrica. In particolare si studiano poi due casi relativi a corpi privi di interazione termoelettrica e a corpi perfetti conduttori-dellelet. tricità. Nel primo caso si mette in evidenza la possibilità di due modi magnetoelastici isotermi e quattro modi magneto-termo-elastici; nel secondo si possono avere modi. di velocità termici e isotermici e modi non di velocità, ma tutti richiedono ulteriori ipotesi semplificatrici quali, ad esempio, l'assenza della corrente di spostamento o una parti. colare direzione di propagazione.

\section{1. - Introduzione.}

Il problema della propagazione di onde piane in magneto-termo-elasticità non è nuovo: il primo lavoro sull'argomento, del quale siamo a conoscenza, relativo a un caso particolare, risale al 1961 ed è dovuto a G. PARIa [1]; altri, sempre su casi particolari, ne sono seguiti successivamente (si veda ad es. [2] e [3]). Nel presente lavoro si affronta la stessa questione facendo uso di un procedimento che ci sembra molto generale, introdotto da A. BAños e applicato alla magnetoidrodinamica [4], [5], [6] e alla magnetoelasticità [7]. Prima di tutto però si ricavano le equazioni del campo: nel n. 2 si scrivono le equazioni di Maxwell mettendo in evidenza l'accoppiamento termoelettrico nell' espressione della densità della corrente elettrica; poi (n. 3) si ricara l'equazione del moto relativa alle piccole oscillazioni elastiche, applicando il teorema delle quantità di moto; infine (n. 4) si giunge all' equazione generalizzata del calore mediante il principio di conservazione dell'energia; sfruttando la legge di Fourier estesa all'interazione termoelettrica e i primi principi della termodinamica ${ }^{1}{ }^{1}$.

(*) Lavoro eseguito nell'ambito dell'attività dei Gruppi di Ricerca Matematici del Consiglio Nazionale delle ricerehe.

(4) Il procedimento, che nei n. 3 e 4 permette di ricarare le due suddette equazioni, non i pare sia stato precedentemente usato nella letteratura riguardante la magneto-termo. elasticità. 
Si passa quindi alla linearizzazione delle equazioni (n. 5) giungendo così ad ottenerne due sole, una vettoriale nella velocità e nella temperatura e una scalare relativa alla diffusione del calore, nella velocità, nella tempera. tura e nella densità della corrente elettrica. Nel n. 6 si considera la propa. gazione di onde piane ammettendo che tutte le grandezze in esame dipendano allo stesso modo dallo spazio e dal tempo mediante il fattore exp $\{\boldsymbol{i}(\boldsymbol{k} \cdot \mathbf{r}-\omega t)\}\left(^{2}\right)$. Introdotta questa ipotesi nelle due equazioni di cui sopra, si sfrutta l'idea del Baños che consiste essenzialmente nel considerare la componente lungo l'asse $\approx$ (che è la direzione del campo magnetico primario $\boldsymbol{B}_{0}$ ), la divergenza e la componente $z$ del rotazionale dell' equazione vettoriale, anzichè studiare le sue tre componenti cartesiane. Queste tre equazioni scalari più quella del calore costituiscono un sistema del quale si cercano, per una assegnata direzione di propagazione, i numeri d'onda corrispondenti a tutti i possibili modi di propagazione per onde piane. Per la complessità delle equazioni si affrontano due casi particolari: nel primo (n. 7) si trasoura l'accoppiamento termoelettrico, nel secondo (n. 8) si considera infinita la conducibilità elettrica. Nel n. 7 dapprima si studiano $\mathbf{i}$ «modi di velocità», quelli cioè per i quali la velocità $v$ è normale al piano formato dal campo magnetico esterno $B_{0}$ e dal vettore di propagazione $\boldsymbol{k}$; questi modi sono possibili solo nel caso isotermo e sono due in tutto. Scegliendo invece per la velocita una particolare combinazione di soluzioni elementari dell' equazione di Helmholtz, si possono avere quattro modi distinti magneto-termo-elastici: questi, in assenza di in. terazione termoelastica, si riducono ai tre modi magnetoelastici già studiati dal BAÑos [7] e a uno puramente termico. L'equazione che fornisce questi quattro modi di propagazione viene poi studiata per $B_{0} \rightarrow 0$ e per $B_{0} \rightarrow \infty$ e alle alte e basse frequenze; sia per $B_{0} \rightarrow 0$ che per $B_{0} \rightarrow \infty$ alle alte frequenze il corpo si comporta come se fosse in assenza di interazione termo. elastica. Nel n. 8, come già si è detto, si considera un corpo perfetto con. duttore dell' elettricità; è opportuno però in questo caso ritoccare le equazioni di Maxwell e quella del calore per giungere al sistema risolutiro di quattro equazioni scalari nella velocità e nella temperatura. Anche qui si considerano dapprima i modi di velocità; a differenza del caso precedente si possono avere modi di velocità termici (ossia con temperatura variabile) nelle ipotesi che sia trascurabile il coefficiente di dilatazione termica o che la direzione di propagazione sia normale al campo magnetico primario. Non si hanno in. vece, senza ulteriori ipotesi, oltre alla $\gamma=\infty$, modi non di velocità del tipo già visto nel n. 7 ; questi modi sono però possibili con altre restrizioni quali l'assenza della corrente di spostamento o dell'interazione termoelettrica gia vista, oppure ammettendo che la propagazione avrenga solo nella direzione

(2) $k$ è il vettore di propagazione, $r$ quello di posizione, (1) indica la pulsazione e $t$ 
del campo magnetico esterno; in quest' ultimo caso si possono avere due modi magneto-termo-elastici che si riducono a uno elastico relativo ad onde di compressione e a uno di natura termoelettrica nel caso in cui non vi sia accoppiamento termoelastico.

\section{2. - Le equazioni del campo elettromagnetico.}

Consideriamo un corpo elastico omogeneo, isotropo, conduttore dell' elettricità e del calore. Indicato con:

$\boldsymbol{H}$ il campo magnetico,

$\boldsymbol{E}$ il campo elettrico,

$B$ l'induzione magnetica,

$\varepsilon$, $\mu$ la costante dielettrica e la permeabilità magnetica,

$\rho_{e}$ la densità volumetrica di carica elettrica,

le equazioni di Maxwell si possono serivere

$$
\begin{gathered}
\nabla \times \boldsymbol{H}=\varepsilon \frac{\partial \boldsymbol{E}}{\partial t}+\boldsymbol{j} \\
\nabla \times \boldsymbol{E}=-\mu \frac{\partial \boldsymbol{H}}{\partial t} \\
\nabla \cdot \boldsymbol{E}=\frac{\rho_{e}}{\varepsilon} \\
\nabla \cdot \boldsymbol{B}=0
\end{gathered}
$$$$
(\boldsymbol{B}=\mu \boldsymbol{H})
$$

dove il vettore $\boldsymbol{j}$, densità di corrente elettrica, è dato da $\left(^{3}\right)$

$$
\dot{j}=\rho_{e} \dot{u}+\gamma(E+\dot{\boldsymbol{u}} \times \boldsymbol{B}-\beta \nabla T)
$$

nella (5) $\gamma$ indica la conducibilita elettrica, $\boldsymbol{u}$ il vettore spostamento elastico, $T$ la temperatura assoluta e $\beta$ è il coefficiente che lega il campo elettrico al gradiente di temperatura. In seguito faremo uso anche del tensore elettromagnetico di Maxwell

$$
\tau_{i k}=\varepsilon\left(E_{i} E_{k}-\frac{1}{2} E^{*} \delta_{i k}\right)+\mu\left(H_{i} H_{k}-\frac{1}{2} H^{2} \delta_{i k}\right)
$$

(3) Per il termine in $\nabla T$ si veda [8], formula (25.2): esso molte in evidenza che, se il corpo è a temperatura non unifome, vi può essere una corrente elettrica anche con un campo nullo. 
dove $\delta_{i k}$ è il tensore di Kronecker, e del vettore di Poynting

$$
\boldsymbol{S}=\boldsymbol{E} \times \boldsymbol{H}
$$

\section{3. - L'equazione delle piccole oscillazioni elastiche.}

Il corpo elastioo può essere soggetto a piccole oscillazioni caratterizzate dal tensore degli storzi $\sigma_{i k}$ e da quello delle deformazioni $\varepsilon_{i k}$ espressi dalle relazioni ( ${ }^{(4)}$

$$
\begin{gathered}
\sigma_{i k}=2 G \varepsilon_{i k}+\left[\lambda e-K \alpha\left(T-T_{0}\right)\right] \delta_{i k}, \\
\varepsilon_{i k}=\frac{1}{2}\left(u_{i / k}+u_{k i k}\right),
\end{gathered}
$$

dove $e \equiv \nabla \cdot u$ è la dilatazione cubica, $\lambda$ e $G$ sono le costanti di Lamè, a ̀̀ il coefficiente di dilatazione termica di volume, $R=\lambda+\frac{2}{3} G$ è il modulo di compressione e $T_{0}$ indica la temperatura assoluta dello stato naturale del corpo.

Per ottenere l'equazione dei piccoli moti elastici, indicando con $\rho$ la densità del mezzo, con $\boldsymbol{F}$ le forze di massa e con $\ddot{\boldsymbol{u}}$ l'accelerazione, scriviamo il teorema della quantità di moto nella forma

$$
\int_{V}\left(\rho \ddot{u}_{i}+\varepsilon \mu \dot{S}_{i}\right) d V=\int_{V} \rho F_{i} d V+\int_{S}\left(\sigma_{i k}+\tau_{i k}\right) n^{k} d S
$$

dove $V$ indica un volume arbitrario all' interno del corpo ed $S$ la superficie che lo racchiude. Per il teorema della divergenza e per l'arbitrarietà del volume considerato, da (10) si ha

$$
\rho \frac{\partial^{2} u_{i}}{\partial t^{2}}=\rho F_{i}+\sigma_{i k}^{l k}+\tau_{i k}^{j k}-\varepsilon \mu \dot{S}_{i}
$$

Se ora si indica con $\nabla \cdot \sigma$ la divergenza del tensore degli sforzi, da (8) si ottiene

$$
\nabla \cdot \sigma=G \nabla^{*} \boldsymbol{u}+(\lambda+G) \nabla \nabla \cdot \mathbf{u}-K \alpha \nabla T,
$$

mentre per il tensore di Maxwell si ha

$$
\nabla \cdot \tau=\varepsilon[\boldsymbol{E} \nabla \cdot \boldsymbol{E}+(\nabla \times \boldsymbol{E}) \times \boldsymbol{E}]+\mu(\nabla \times \boldsymbol{H}) \times \boldsymbol{H} .
$$

(4) Per queste si veda, ad. es., [9] pag. 39. 
Per (1), (2) e (3) la (13) si può scrivere

(14) $\nabla \cdot \tau=\rho_{e} \boldsymbol{E}-\varepsilon \mu \frac{\partial \boldsymbol{H}}{\partial t} \times \boldsymbol{E}+\mu\left(\boldsymbol{j}+\varepsilon \frac{\partial \boldsymbol{E}}{\partial t}\right) \times \boldsymbol{H}=\rho_{e} \boldsymbol{E}+\mu \boldsymbol{j} \times \boldsymbol{H}+\mu \varepsilon \frac{\partial}{\partial t}(\boldsymbol{E} \times \boldsymbol{H})$.

La (11) quindi, in virtù di (12) e (14), si può mettere, per comodità di calcolo, nella forma vettoriale seguente

$$
\rho \frac{\partial^{2} \boldsymbol{u}}{\partial t^{2}}=\rho \boldsymbol{F}+G \nabla^{2} \mathbf{u}+(\lambda+G) \nabla \nabla \cdot \boldsymbol{u}-K \alpha \nabla \boldsymbol{T}+\rho_{e} \boldsymbol{E}+\boldsymbol{j} \times \boldsymbol{B} .
$$

Se poi si introduce il vettore

$$
\boldsymbol{f}=\rho_{e} \boldsymbol{E}+\boldsymbol{j} \times \boldsymbol{B}
$$

che rappresenta tutte le forze elettromagnetiche e che, per quel che precede, è espresso anche da

$$
f_{i}=\tau_{i k}^{l k}-\varepsilon \mu \dot{S}_{i}
$$

l'equazione del moto (11) diventa

$$
\rho \frac{\partial^{2} u_{i}}{\partial t^{2}}=\rho F_{i}+\sigma_{i k}^{k}+f_{i}
$$

\section{4. - L'equazione del calore.}

Applicando il principio della conservazione dell' energia si ricava l' equazione della diffusione del calore per i problemi magneto-termo-elastici. Siano:

$U$ l'energia interna specifica (per unità di volume),

q la densità di corrente termica espressa dalla legge di Fourier generalizzata $\left({ }^{5}\right)$

$$
q_{i}=-x T_{i i}+\pi j_{i}
$$

dove $x$ è il coefficiente di conducibilità termica e $\pi$ quello di Peltier che lega la corrente termica a quella elettrica $\left(^{6}\right)$; siano poi: $w=1 / 2\left(\varepsilon E^{2}+\mu H^{2}\right)$ l'energia elettromagnetica per unità di volume, $Q^{*}$ la quantità di calore generata da sorgenti nell' unità di tempo e per unità di volume.

(5) Per questa legge si veda la formulazione adottata da K KLISKI in [13], formula (2.11), relativamente al caso isotropo omogeneo.

(6) Nel caso di un mezzo anisotropo nella (19) compare, anzichè $\pi j_{i}, \pi_{i k} j_{k}$, dove $\pi_{i k}$ è il tensore di Peltier; la variazione da posto a posto di $\pi_{i k}$ è responsabile della produzione del calore di Peltier, mentre quella di $j$ genera il calore di Bridgman (si veda [14]). 
Il teorema della conservazione dell'energia allora si può scrivere:

$$
\int_{V}\left(\rho v_{i} \dot{v}^{i}+\dot{U}+\dot{v}\right) d V=\int_{V} \rho F_{i} v^{i} d V+\int_{V} Q^{*} d V+\int_{S}\left(\sigma_{i k} v^{k}-q_{i}-S_{i}\right) n^{i} d S
$$

dove con $v=\dot{u}$ si è indicato la velocita di spostamento elastico. Per il teo. rema della divergenza il secondo membro di (20) diventa

$$
\int_{V}\left(\rho F_{i} v^{i}+Q^{*}+\sigma_{i k} v^{k / i}+\sigma_{i k}^{i i} v^{k}-q_{i}^{i i}-S_{i}^{\prime i}\right) d V
$$

Quindi dalla (20) segue

$$
\rho v_{i} \dot{v}^{i}+\dot{U}+\dot{w}=\rho F_{i} v^{i}+\sigma_{i k} v^{k i / i}+\sigma_{i k}^{/ i} v^{k}-q_{i}^{/ i}-S_{i}^{i i}+Q^{*} .
$$

Tenendo conto dell' equazione del moto (18) e della (19), la (21) si può scrivere

$$
\dot{U}+\dot{w}=\sigma_{i k} v^{k / i}-f_{i} v^{i}+x \nabla^{2} T-\pi \nabla \cdot j-S_{i}^{i}+Q^{*} .
$$

Ora dalla (16) segue

$$
\boldsymbol{f} \cdot \boldsymbol{v}=\rho_{e} \boldsymbol{E} \cdot \boldsymbol{v}-\boldsymbol{j} \cdot(\boldsymbol{v} \times \boldsymbol{B}) ;
$$

introducendo la densità di corrente di conduzione

$$
\boldsymbol{J}=\boldsymbol{j}-\rho_{e} \boldsymbol{v}=\gamma(\boldsymbol{E}+\boldsymbol{v} \times \boldsymbol{B}-\beta \nabla T),
$$

la (23), tenendo presenti le equazioni di Maxwell, diventa

$$
\begin{gathered}
\boldsymbol{f} \cdot \boldsymbol{v}=\rho_{e} \boldsymbol{E} \cdot \boldsymbol{v}-\boldsymbol{j} \cdot\left(\frac{\boldsymbol{J}}{\gamma}-\boldsymbol{E}+\beta \nabla T\right)=\rho_{e} \boldsymbol{E} \cdot \boldsymbol{v}-\boldsymbol{j} \cdot \frac{\boldsymbol{J}}{\gamma}+\boldsymbol{j} \cdot \boldsymbol{E}-\beta \boldsymbol{j} \cdot \nabla T= \\
=\rho_{e} \boldsymbol{E} \cdot \boldsymbol{v}-\left(\boldsymbol{J}+\rho_{e} \boldsymbol{v}\right) \cdot \frac{\boldsymbol{J}}{\gamma}+\left(\nabla \times \boldsymbol{H}-\varepsilon \frac{\partial \boldsymbol{E}}{\partial t}\right) \cdot \boldsymbol{E}-\beta \boldsymbol{j} \cdot \nabla T= \\
=\rho_{e} \boldsymbol{E} \cdot \boldsymbol{v}-\frac{J^{2}}{\gamma}-\rho_{e} \boldsymbol{v} \cdot(\boldsymbol{E}+\boldsymbol{v} \times \boldsymbol{B}-\beta \nabla T)+\nabla \times \boldsymbol{H} \cdot \boldsymbol{E}-\frac{1}{2} \varepsilon \frac{\partial E^{2}}{\partial t}-\beta \boldsymbol{j} \cdot \nabla \boldsymbol{T}= \\
=-\frac{J^{2}}{\gamma}+\beta_{e} \boldsymbol{v} \cdot \nabla \boldsymbol{T}-\nabla \cdot(\boldsymbol{E} \times \boldsymbol{H})+\nabla \times \boldsymbol{E} \cdot \boldsymbol{H}-\frac{1}{2} \varepsilon \frac{\partial E^{2}}{\partial t}-\beta \boldsymbol{j} \cdot \nabla T= \\
=-\frac{J^{2}}{\gamma}-\beta \boldsymbol{J} \cdot \nabla T-\nabla \cdot \boldsymbol{S}-\frac{1}{2} \frac{\partial}{\partial t}\left(\varepsilon E^{2}+\mu H^{2}\right) .
\end{gathered}
$$


In conclusione si ha

$$
f_{i} v^{i}=-\frac{J^{q}}{\gamma}-\beta J \cdot \nabla T-w-S_{i}^{i}
$$

la (22) quindi assume la forma

$$
\frac{\partial U}{\partial t}=\sigma_{i k} v^{k / i}+\frac{J^{2}}{\gamma}+\beta \boldsymbol{J} \cdot \nabla T+\chi \nabla^{2} T-\pi \nabla \cdot \boldsymbol{j}+Q^{*} .
$$

Esplicitiamo ora il primo membro di (26); potendo scrivere ( $\left.{ }^{7}\right)$

$$
U=U\left(\varepsilon_{i k}, T\right),
$$

ne segue

$$
\frac{\partial U}{\partial t}=\frac{\partial U}{\partial T} \frac{\partial T}{\partial t}+\frac{\partial U}{\partial \varepsilon_{i k}} \frac{\partial \varepsilon_{i k}}{\partial t}
$$

Ora, indicando con $\delta Q$ il calore assorbito nel tempo $d t$ dall'unità di volume, il primo prinejpio della termodinamica permette di serivere

$$
\delta Q=\frac{\partial U}{\partial T} d T+\frac{\partial U}{\partial \varepsilon_{i k}} d \varepsilon_{i k}-\sigma_{i k} d \varepsilon^{i / \varepsilon}
$$

o anche, introducendo l'entropia specifica (per unità di volume) $s=s\left(\varepsilon_{i k}, T\right)$,

$$
d U=T d s+\sigma_{i k} d \varepsilon^{i k}
$$

ossia

$$
d U=T \frac{\partial s}{\partial T} d T+\left[\sigma_{i k}+T \frac{\partial s}{\partial \varepsilon^{i k}}\right] d \varepsilon^{i k}
$$

Dalla (28), considerando trasformazioni a deformazione costante e introducendo il calore specifico a deformazione costante $C_{\varepsilon}$, definito da $\left(^{8}\right)$

$$
(\delta Q)_{\varepsilon}=\rho C_{\varepsilon} d T
$$

si ottiene

$$
\rho C_{\varepsilon}=\frac{\partial U}{\partial T}
$$

da (30) invece si ha

$$
\frac{\partial U}{\partial \varepsilon^{i k}}=\sigma_{i k}+T \frac{\partial s}{\partial \varepsilon^{i k}}
$$

(7) Per questa ipotesi e per la relazione (28) si veda, ad esempio, [9] pag. 39.

(8) Si veda ad esempio, [10], pag. 194 e seg. 
Inoltre, essendo $U$ una funzione di stato, il secondo membro di (30) deve essere un differenziale esatto e perciò si ricava

$$
\frac{\partial s}{\partial \varepsilon^{i k}}=-\frac{\partial \sigma_{i k}}{\partial T}
$$

quindi la (32) diventa

$$
\frac{\partial U}{\partial \varepsilon^{i k}}=\sigma_{i k}-T \frac{\partial \sigma_{i k}}{\partial T}
$$

e, per la (8),

$$
\frac{\partial U}{\partial \varepsilon^{i k}}=\sigma_{i k}+K \alpha T \delta_{i k}
$$

In conclusione la (27) si può scrivere

$$
\begin{aligned}
\frac{\partial U}{\partial t}= & \rho C_{\varepsilon} \frac{\partial T}{\partial t}+\sigma_{i \hbar} \dot{\varepsilon}^{i k}+K \alpha T \delta_{i k} \dot{\varepsilon}^{i k}= \\
& =\rho C_{\varepsilon} \frac{\partial T}{\partial t}+\sigma_{i k} \dot{\varepsilon}^{i k}+K \alpha T \dot{e} .
\end{aligned}
$$

Infine la (26), per la (36) e ricordando anche la $(9$, diventa

$$
\rho C_{\varepsilon} \frac{\partial T}{\partial t}+K \alpha T \nabla \cdot \boldsymbol{v}=\chi \nabla^{2} T+\frac{J^{2}}{\gamma}+\beta \boldsymbol{J} \cdot \nabla T-\pi \nabla \cdot \boldsymbol{j}+Q^{*} .
$$

Alla (37) si pnò dare un'altra forma leggermente diversa; introducendo il calore specifico a stress costante dato da, [10],

$$
(\delta Q)_{\sigma}=\rho C_{\sigma} d T
$$

la (28) può scriversi

$$
\rho C_{\sigma} d T=\rho C_{\varepsilon} d T+\left[\frac{\partial U}{\partial \varepsilon^{i k}}-\sigma_{i k}\right] d \varepsilon^{i k}
$$

da cui segue

$$
\rho\left(C_{\sigma}-C_{\varepsilon}\right)=\left[\frac{\partial U}{\partial \varepsilon^{i k}}-\sigma_{i k}\right]\left(\frac{\partial \varepsilon^{i k}}{\partial T}\right)_{\sigma}
$$

o anche, per la (34)

$$
p\left(C_{\sigma}-C_{\varepsilon}\right)=-T \frac{\partial \sigma_{i k}}{\partial T}\left(\frac{\partial \varepsilon^{i k}}{\partial T}\right)_{\sigma}
$$

dove le derivate $\frac{\partial \varepsilon^{i k}}{\partial T}$ sono fatte per $\mathrm{i}$ valori costanti delle $\sigma_{i k}$. 
Ora per le (8) e per le relazioni inverse che da esse si possono ottenere

$$
\varepsilon_{i k}=\frac{\alpha}{3} T \delta_{i k}+\frac{1}{2 G}\left[\sigma_{i k}-\frac{\lambda}{3 K} \sigma_{r}^{\cdot r} \delta_{i k}\right]
$$

la (41) fornisce

$$
\rho\left(C_{\sigma}-C_{\varepsilon}\right)=K \alpha^{2} T
$$

Sostituendo quest' ultima in (37) si ottiene

$$
\rho C_{\varepsilon} \frac{\partial T}{\partial t}+\rho \frac{C_{\sigma}-C_{\varepsilon}}{\alpha} \nabla \cdot v=x \nabla^{2} T+\frac{J^{2}}{\gamma}+\beta J \cdot \nabla T-\pi \nabla \cdot j+Q^{*}
$$

Il primo termine a primo membro esprime l'incremento di calore nell' unità di tempo e di volume a seguito della variazione di $T$, il secondo termine quello dovuto alla variazione della dilatazione cubica; a secondo membro si ha il calore dovuto all'ordinaria conduzione termica, quello sviluppato per effetto Joule, proporzionale al quadrato della densità della corrente di conduzione, e quello $Q^{*}$ generato dalle sorgenti di calore. I due termini rimanenti esprimono, il primo, il calore prodotto per effetto Thomson (che è proporzionale, a differenza di quello di Joule, alla densità della corrente di conduzione e quindi cambia segno se si inverte il senso di tale corrente), il secondo il calore sviluppato per effetto BRIDGMaN [14] che dipende dalla variazione da posto a posto della densità della corrente elettrica. Questo calore, se si trascura la corrente di spostamento per cui è $\nabla \cdot \boldsymbol{j}=0$, si riscontra solo nei mezzi anisotropi e si manifesta alla superficie dei conduttori, dove la densità $j$ diminuisce rapidamente oppure alla superficie di separazione di due conduttori diversi, dove il vettore $\boldsymbol{j}$ cambia

di direzione. Nel nostro caso, essendo invece $\nabla \cdot j=\nabla \cdot\left(\varepsilon \frac{\partial E}{\partial t}\right)=\varepsilon \frac{\partial \rho_{e}}{\partial t}$, tale produzione di calore è dovuta alla variazione da posto a posto della corrente di spostamento, o, se si vuole, alla variazione nel tempo della carica elettrica di volume.

\section{5. - Linearizzazione delle equazioni.}

Consideriamo dapprima l'equazione del calore nella forma (43) ammettendo, per semplicità, che non vi siano delle sorgenti di calore; introducendo la temperatura relativa allo stato naturale del corpo

$$
\Theta=T-T_{0}
$$


la (43) diventa

$$
\rho C_{\varepsilon} \frac{\partial \Theta}{\partial t}+\rho \frac{C_{\sigma}-C_{\varepsilon}}{\alpha} \nabla \cdot v=x \nabla^{2} \Theta+\frac{J^{2}}{\gamma}+\beta \boldsymbol{J} \cdot \nabla \Theta-\pi \nabla \cdot \boldsymbol{j} .
$$

Trascurando ora i termini di grado superiore al primo si ottiene

$$
\rho O_{\varepsilon} \frac{\partial \Theta}{\partial t}+\rho \frac{C_{\sigma}-C_{\varepsilon}}{\alpha} \nabla \cdot v=x \nabla^{2} \Theta-\pi \nabla \cdot j
$$

che è appunto l'equazione della diffusione del calore per.i problemi magne. to-termo-elastici in forma lineare. Analogamente dalla (37) si ricava $\left({ }^{9}\right)$

$$
\rho C_{\varepsilon} \frac{\partial \Theta}{\partial t}+K \alpha T_{0} \nabla \cdot v=\chi \nabla^{2} \Theta--\pi \nabla \cdot j
$$

Per linearizzare le altre equazioni $\left({ }^{10}\right)$, seriviamo l'induzione magnetica nella forma

$$
\boldsymbol{B}=\boldsymbol{B}_{0}+\mu \boldsymbol{h}
$$

dove $\boldsymbol{B}_{0}$ rappresenta il campo esterno, che supponiamo costante, $\boldsymbol{\theta} \boldsymbol{h}$ il campo indotto. Consideriamo poi il rotazionale della (2), tenendo presente la (1),

$$
\nabla \times \nabla \times \boldsymbol{E}=-\mu \frac{\partial}{\partial t}(\nabla \times \boldsymbol{H})=-\mu \frac{\partial}{\partial t}\left(\varepsilon \frac{\partial \boldsymbol{E}}{\partial t}+\boldsymbol{j}\right)
$$

da eni segue

$$
\nabla^{2} \boldsymbol{E}-\mu \varepsilon \frac{\partial^{2} \boldsymbol{E}}{\partial t^{2}}-\mu \frac{\partial j}{\partial t}=\nabla \nabla \cdot E
$$

Dalla (5) si ottiene

$$
\gamma \nabla \nabla \cdot \boldsymbol{E}=\nabla \nabla \cdot \boldsymbol{j}-\nabla \nabla \cdot\left(\rho_{\theta} \boldsymbol{v}\right)-\gamma \nabla \nabla \cdot(\boldsymbol{v} \times \boldsymbol{B})+\beta \gamma \nabla \nabla \geqslant T
$$

mentre da (1), tenendo presente la (2) e la (1) stessa, si ha

$$
\begin{gathered}
\nabla \nabla \cdot j=- \\
=\nabla \nabla \cdot \frac{\partial E}{\partial t}=-\varepsilon \nabla^{2} \frac{\partial E}{\partial t}-\varepsilon \nabla \times \nabla \times \frac{\partial E}{\partial t}= \\
=-\varepsilon \nabla^{2} \frac{\partial E}{\partial t}+\varepsilon \mu \nabla \times \frac{\partial^{2} \boldsymbol{H}}{\partial t^{2}}= \\
=-\varepsilon \nabla^{2} \frac{\partial E}{\partial t}+\varepsilon \mu \frac{\partial^{2}}{\partial t^{2}}\left(j+\varepsilon \frac{\partial E}{\partial t}\right) .
\end{gathered}
$$

(9) Questa equazione e la (45) si confrontino, ad esempio, con la (1.3) di [11] e la (2.3) di [12].

(10) Per questo procedimento si veda [4]. 
Sostituiamo ora (49) e (50) nella (48)

$$
\begin{gathered}
\gamma \nabla^{2} \boldsymbol{E}-\gamma \mu \varepsilon \frac{\partial^{2} \boldsymbol{E}}{\partial t^{2}}-\gamma \mu \frac{\partial \boldsymbol{j}}{\partial t}=-\varepsilon \nabla^{2} \frac{\partial \boldsymbol{E}}{\partial t}+\mu \varepsilon \frac{\partial \boldsymbol{j}}{\partial t}+ \\
+\mu \varepsilon^{2} \frac{\partial^{3} \boldsymbol{E}}{\partial t^{3}}-\nabla \nabla \cdot\left(\rho_{e} v\right)-\gamma \nabla \nabla \cdot(\boldsymbol{v} \times \boldsymbol{B})+\beta \gamma \nabla \nabla^{2} T,
\end{gathered}
$$

ossia ricordando anche la (44),

$$
\begin{gathered}
\left(\gamma+\varepsilon \frac{\partial}{\partial t}\right)\left(\nabla^{2}-\varepsilon \mu \frac{\partial^{2}}{\partial t^{2}}\right) \boldsymbol{E}+\gamma \nabla \nabla \cdot(\boldsymbol{v} \times \boldsymbol{B})= \\
=\mu \frac{\partial}{\partial t}\left(\gamma+\varepsilon \frac{\partial}{\partial t}\right) \boldsymbol{j}-\nabla \nabla \cdot\left(\rho_{e} \boldsymbol{v}\right)+\beta \gamma \nabla \nabla^{2} \Theta .
\end{gathered}
$$

Moltiplichiamo ora vettorialmente a destra ambo i membri di (52) per il vettore costante $\boldsymbol{B}_{0}$

$$
\begin{aligned}
& \left(\gamma+\varepsilon \frac{\partial}{\partial t}\right)\left(\nabla^{2}-\varepsilon \mu \frac{\partial^{2}}{\partial t^{2}}\right)\left(\boldsymbol{E} \times \boldsymbol{B}_{0}\right)+\gamma[\nabla \nabla \cdot(\boldsymbol{v} \times \boldsymbol{B})] \times \boldsymbol{B}_{0}= \\
= & \mu \frac{\partial}{\partial t}\left(\gamma+\varepsilon \frac{\partial}{\partial t}\right)\left(\boldsymbol{j} \times \boldsymbol{B}_{0}\right)-\left[\nabla \nabla \cdot\left(\rho_{e} \boldsymbol{v}\right)\right] \times \boldsymbol{B}_{0}+\beta \gamma\left[\nabla \nabla^{2} \Theta\right] \times \boldsymbol{B}_{0} .
\end{aligned}
$$

Introducendo la (46) e la (3) nella (16) si ha

$$
\boldsymbol{f}=\varepsilon \boldsymbol{E} \nabla \cdot \boldsymbol{E}+\boldsymbol{j} \times \boldsymbol{B}_{0}+\mu \boldsymbol{j} \times \boldsymbol{h}
$$

da questa segue

$$
\boldsymbol{j} \times \boldsymbol{B}_{0}=\boldsymbol{f}-\nabla \cdot \tau+\varepsilon \mu \frac{\partial}{\partial t}(\boldsymbol{E} \times \boldsymbol{h})
$$

dove qui $\tau$ indica il tensore elettromagnetico di Maxwell per il campo in. dotto, ossia

$$
\tau_{i k}=\varepsilon\left(E_{i} E_{k}-\frac{1}{2} E^{\prime} \delta_{i k}\right)+\mu\left(h_{i} h_{k}-\frac{1}{2} h^{2} \delta_{i k}\right)
$$

D'altra parte dalla (5) si ha anche

$$
\boldsymbol{E}=\frac{1}{\gamma} \boldsymbol{j}-\frac{1}{\gamma} \rho_{e} \boldsymbol{v}-\boldsymbol{v} \times \boldsymbol{B}+\beta \nabla \Theta
$$

e quindi

$$
E \times B_{0}=\gamma^{-1} \boldsymbol{j} \times B_{0}-\gamma^{-1} \rho_{e} v \times B_{0}-(\boldsymbol{v} \times B) \times B_{0}+\beta \nabla \Theta \times B_{0}
$$


e ancora, per la (54)

$$
\boldsymbol{E} \times \boldsymbol{B}_{0}=\boldsymbol{\gamma}^{-1}\left[\boldsymbol{f}-\nabla \cdot \tau+\varepsilon \mu \frac{\partial}{\partial t}(\boldsymbol{E} \times \boldsymbol{h})\right]-\gamma^{-1} \rho_{e} \boldsymbol{v} \times \boldsymbol{B}_{0}-(\boldsymbol{v} \times \boldsymbol{B}) \times \boldsymbol{B}_{0}+\beta \nabla \Theta \times \boldsymbol{B}_{0} .
$$

Sostituiamo ora $(54)$ e $(55)$ in $(53)$

$$
\begin{gathered}
\left(\boldsymbol{\gamma}+\varepsilon \frac{\partial}{\partial t}\right)\left(\nabla^{2}-\varepsilon \mu \frac{\partial^{2}}{\partial t^{2}}\right)\left\{\gamma^{-1}\left[\boldsymbol{f}-\nabla \cdot \tau+\varepsilon \mu \frac{\partial}{\partial t}(\boldsymbol{E} \times \boldsymbol{h})-\rho_{e} \boldsymbol{v} \times \boldsymbol{B}_{0}\right]-\right. \\
\left.-(\boldsymbol{v} \times \boldsymbol{B}) \times \boldsymbol{B}_{0}+\beta \nabla \Theta \times \boldsymbol{B}_{0}\right\}+\gamma[\nabla \nabla \cdot(\boldsymbol{v} \times \boldsymbol{B})] \times \boldsymbol{B}_{0}= \\
=\mu \frac{\partial}{\partial t}\left(\gamma+\varepsilon \frac{\partial}{\partial t}\right)\left[\boldsymbol{f}-\nabla \cdot \tau+\varepsilon \mu \frac{\partial}{\partial t}(\boldsymbol{E} \times \boldsymbol{h})\right]-\left[\nabla \nabla \cdot\left(\rho_{e} \boldsymbol{v}\right)\right] \times \boldsymbol{B}_{0}+ \\
+\beta \gamma\left[\nabla \nabla^{2} \Theta\right] \times \boldsymbol{B}_{0} .
\end{gathered}
$$

Della (56) prendiamo in considerazione la forma linearizzata seguente

$$
\begin{aligned}
& \left(\gamma+\varepsilon \frac{\partial}{\partial t}\right)\left(\nabla^{2}-\varepsilon \mu \frac{\partial^{2}}{\partial t^{2}}\right)\left[\gamma^{-1} \boldsymbol{f}-\left(\boldsymbol{v} \times \boldsymbol{B}_{0}\right) \times \boldsymbol{B}_{0}+\beta \nabla \Theta \times \boldsymbol{B}_{0}\right]= \\
& =\mu\left(\gamma+\varepsilon \frac{\partial}{\partial t}\right) \frac{\partial f}{\partial t}-\gamma\left[\nabla \nabla \cdot\left(\boldsymbol{v} \times \boldsymbol{B}_{0}\right)\right] \times \boldsymbol{B}_{0}+\beta \gamma\left[\nabla \nabla^{2} \theta\right] \times \boldsymbol{B}_{0},
\end{aligned}
$$

dove, per la (12) e la (18) in cui si trascurino le forze di volume $p \boldsymbol{F}$, è

$$
\frac{\partial f}{\partial t}=\rho \frac{\partial^{2} v}{\partial t^{2}}-\rho\left(V_{o}^{2}-V_{d}^{2}\right) \nabla \nabla \cdot v-\rho V_{d}^{2} \nabla^{2} v+K \alpha \frac{\partial}{\partial t} \nabla \Theta ;
$$

nella (58) si sono introdotte le velocità di compressione e di distorsione date da

$$
V_{c}^{2}=\frac{\lambda+2 G}{\rho}, \quad V_{d}^{2}=\frac{G}{\rho} .
$$

La $\left(45^{\prime}\right)$ e la (57) sono le equazioni su cui ci baseremo per studiare le onde piane.

\section{6. - Onde piane.}

Supponiamo che la temperatura e le componenti cartesiane dei vettori de] campo presentino la stessa dipendenza dal tempo e dallo spazio espressa dal fattore adimensionale

$$
\psi(\boldsymbol{r}, t)=\exp \{i(\boldsymbol{k} \cdot \boldsymbol{r}-\omega t)\}
$$


dove $\omega$ è la pulsazione assegnata delle oscillazioni armoniche nel tempo e $\boldsymbol{k}$, in generale complesso, $\partial$ il vettore di propagazione. La funzione $\psi$ soddisfa all' equazione di. Helmholtz

$$
\left(\nabla^{2}+k^{2}\right) \psi=0
$$

Scegliamo poi l'asse $z$ parallelo all'induzione magnetica esterna $B_{0}$ in modo che sia

$$
\boldsymbol{B}_{0}=B_{0} i_{3}
$$

essendo $i_{a}$ il versore dell'asse $z$; allora siccome tale asse costituisce per i fenomeni un asse di simmetria, si può porre in generale

$$
k=k_{n}=k_{x} i_{1}+k_{z} i_{3}
$$

dove $k_{x}$ e $k_{z}$ costituiscono il numero d'onda trasversale e longitudinale rispettivamente. Introduciamo ora per le velocità le soluzioni elementari definite dalle relazioni, [4],

$$
v_{1}=v_{0} \psi i_{2}, \quad v_{2}=n \times v_{1}=v_{0} \psi n \times i_{2}, \quad v_{3}=v_{0} \psi n,
$$

dove $\psi$ è data dalla $(60)$ e $v_{0}$ è un'ampiezza arbitraria di velocità; i tre vettori (63) sono soluzioni dell' equazione vettoriale $\left(\nabla^{2}+k^{2}\right) v=0$ e i primi due sono solenoidali ( $\left.{ }^{1 i}\right)$,

$$
\boldsymbol{k} \cdot \boldsymbol{v}_{1}=0, \quad \boldsymbol{k} \cdot \boldsymbol{v}_{2}=0,
$$

mentre il terzo e irrotazionale

$$
\boldsymbol{k} \times \boldsymbol{v}_{3}=0
$$

\section{Poniamo ora}

$$
v=v_{t}+v_{z} i_{3} \text { e } \nabla=\nabla_{t}+i_{3} \frac{\partial}{\partial_{\varepsilon}}
$$

mettendo in evidenza la parte lungo l'asse $z$ e quella ad esso normale; se poi indichiamo con $-i \omega$ la derivata $\frac{\partial}{\partial t}$, la $(57)$, moltiplicata per l'unità im. maginaria $i$, si può scrivere

$$
\begin{gathered}
(\omega \varepsilon+i \gamma)\left(\nabla^{2}+\omega^{2} \mu \varepsilon\right)\left[\gamma^{-1} \boldsymbol{f}+B_{0}{ }^{2} v_{t}+\beta B_{0} \nabla_{t} \theta \times i_{3}\right]= \\
=-i \omega \mu(\omega \varepsilon+i \gamma) f+i \gamma B_{0}{ }^{2}\left(\nabla_{t}{ }^{2} v_{t}-\nabla_{t} \nabla_{t} \cdot v_{t}\right)+i \gamma \beta B_{0}\left(\nabla_{t} \nabla^{2} \theta\right) \times i_{3} .
\end{gathered}
$$

(14) In base alla (60) l'operatore $\nabla$ vale $i h$. 
Analogamente da (58) si ottiene

$$
i \omega f=\rho \omega^{2} v+\rho\left(V_{c}^{2}-V_{d}^{2}\right) \nabla \nabla \cdot v+\rho V_{d}^{2} \nabla^{2} v+i \omega K \alpha \nabla \Theta .
$$

Introducendo infine $(66)$ in $(65)$ si ricava

$$
\begin{aligned}
&(\omega \varepsilon+i \gamma)\left(\nabla^{2}+\omega^{2} \mu \varepsilon\right)\left\{\frac { 1 } { i \omega \gamma } \left[\rho \omega^{2} v+\rho\left(V_{c}{ }^{2}-V_{d}{ }^{2}\right) \nabla \nabla \cdot v+\rho V_{d}{ }^{2} \nabla^{2} \boldsymbol{v}+\right.\right. \\
&\left.+i \omega K \alpha \nabla \Theta]+B_{0}{ }^{2} \boldsymbol{v}_{t}+\beta B_{0} \nabla_{t} \Theta \times \boldsymbol{i}_{3}\right\}=-\mu(\omega \varepsilon+i \gamma)\left[\rho \omega^{2} \boldsymbol{v}+\right. \\
&\left.+\rho\left(V_{c}{ }^{2}-V_{d}{ }^{2}\right) \nabla \nabla \cdot \boldsymbol{v}+\rho V_{d^{2}} \nabla^{2} \boldsymbol{v}+i \omega K \alpha \nabla \Theta\right]+i \gamma B_{0}{ }^{2}\left(\nabla_{t}{ }^{2} \boldsymbol{v}_{t}-\nabla_{t} \nabla_{t} \cdot \boldsymbol{v}_{t}\right)+ \\
&+i \gamma \beta B_{0}\left(\nabla t \nabla^{2} \Theta\right) \times \boldsymbol{i}_{3} .
\end{aligned}
$$

Di questa equazione, sempre seguendo Baros, [5], consideriamo la componente lungo l'asse $z$, la divergenza e la componente $z$ del rotazionale; si ottengono così le tre seguenti equazioni scalari $\left({ }^{12}\right)$ :

$$
\begin{gathered}
\left.+k_{a}{ }^{2}\left(\nabla^{2}+k_{d}{ }^{2}\right)\left(\nabla^{2}+k_{c}{ }^{2}\right)\right] \nabla \cdot v=-i \frac{K \alpha}{\rho \omega} k_{c}{ }^{2}\left[\left[\left(\nabla^{2}+k_{L}{ }^{2}\right)\left[k_{d}{ }^{2}-i a\left(\nabla^{2}+k_{d}{ }^{2}\right)\right]+\right.\right. \\
\left.+k_{a}{ }^{2}\left(\nabla^{2}+k_{d}{ }^{2}\right) ! \nabla^{2}-k_{d}{ }^{2}\left(\nabla^{2}+k_{L}{ }^{2}\right) \nabla t_{t}{ }^{2}\right] \Theta
\end{gathered}
$$

$$
\begin{gathered}
\left\{\left(\nabla^{2}+k_{L}{ }^{2}\right)\left[k_{d}^{2}-i a\left(\nabla^{2}+k_{d}^{2}\right)\right]+{k_{a}}^{2}\left(\nabla^{2}+k_{d}{ }^{2}\right)-k_{d}{ }^{2}(1-i b)^{-1} \nabla_{t}^{2}\left(\left(\nabla \times v \cdot i_{3}\right)=\right.\right. \\
=\frac{k_{d}^{2} \beta}{B_{0}}\left[\left(\nabla^{2}+k_{L}{ }^{2}\right)-(1-i b)^{-1} \nabla^{2}\right] \nabla_{t}^{2} \theta
\end{gathered}
$$

dove si è indicato:

$k_{L}{ }^{2}=\omega^{2} \mu \varepsilon \quad$ il numero d'onda associato alla velocità della luce

$$
k_{d}{ }^{2}=\omega^{2} / V_{d}^{2} \quad 》 \quad 》 \quad 》 \quad 》 \quad \text { di fase di distorsione }
$$$$
k_{c}^{2}=\omega^{2} / V_{c}^{2} \gg \gg \gg \gg \gg \gg \text { di compressione }
$$$$
k_{a}{ }^{2}=\omega^{2} \mu \rho / B_{0}{ }^{2} \gg \gg \quad \gg \quad \gg \quad \gg \gg \text { di Alfven; }
$$

$a=\omega \rho / \gamma B_{0}{ }^{2}$ e $b=\omega \varepsilon / \gamma$ sono due parametri adimensionali che vanno a zero nel caso di infinita conducibilità elettrica $(\gamma=\infty)$.

Si tratta ora, per una assegnata direzione di propagazione individuata

(12) Pe ottener la (69) si tiene conto anche della (68). 
dall' angolo $\$$, angolo ohe il vettore di propagazione $\boldsymbol{k}$ forma con l'asse $z$, di trovare i numeri d'onda $k$ che corrispondono a dei modi possibili di propagazione. Data la complessità delle equazioni in questione ci limiteremo però ad esaminare alcuni casi particolari.

7. - I caso: sia trascurabile l'accoppiamento termnelettrico $(\beta=0, \pi=0)$.

Supponiamo che gli effetti termoelettrici siano trascurabili rispetto a quelli termoelastici e magnetoelastici in modo da poter considerare nulli $i$ coefficienti $\beta$ e $\pi\left({ }^{13}\right)$; in tal caso l'equazione del calore, nella forma (45'). tenendo conto dell'ipotesi (60) si può scrivere

$$
\left(i \omega \rho C_{\varepsilon}-x k^{2}\right) \Theta=K \alpha T_{0} \nabla \cdot v
$$

mentre da $(68),(69)$ e $(70)$ si ha $\left({ }^{1.4}\right)$

$$
\begin{gathered}
k_{c}^{2}\left(k_{d}^{2}-k^{2}\right) v_{z}=-\left(k_{d}^{2}-k_{e}^{2}\right) \frac{\partial}{\partial z}(\nabla \cdot v)-i \frac{K \alpha}{\rho \omega} k_{e}^{2} k_{d}^{2} \frac{\partial \Theta}{\partial z}, \\
{\left[( k _ { L } ^ { 2 } - k ^ { 2 } ) \left\{\left(k_{d}^{2}-k_{c}^{2}\right) \frac{\partial^{2}}{\partial z^{2}}+\left(k_{d}^{2}-k^{2}\right)\left[k_{c}^{2}-i a\left(k_{c}^{2}-k^{2}\right)\right]+\right.\right.} \\
\left.k_{a}^{2}\left(k_{d}^{2}-k^{2}\right)\left(k_{c}^{2}-k^{2}\right)\right](\nabla \cdot v)=i \frac{K \alpha}{\rho \omega} k_{c}^{2}\left[\left(k_{L}^{2}-k^{3}\right)\left[k_{d}^{2}-i a\left(k_{d}^{2}-k^{2}\right)\right]+\right. \\
\left.\left.+k_{a}^{2}\left(k_{d}^{2}-k^{2}\right)\right\} k^{2}-k_{d}^{2}\left(k_{L}^{2}-k^{2}\right) k^{2} \sin ^{2} \theta\right] \Theta, \\
\left\{\left(k_{L}^{2}-k^{2}\right)\left[k_{d}^{2}-i a\left(k_{d}^{2}-k^{2}\right)\right]+k_{a}^{2}\left(k_{d}^{2}-k^{2}\right)+k_{d}^{2}(1-i b)^{-1} k^{2} \sin ^{2} \theta\right\}\left(\nabla \times v \cdot \dot{i}_{3}\right)=0 .
\end{gathered}
$$

Se si sceglie per la velocita il valore $\left(63_{1}\right)$

$$
v=v_{1}
$$

si ottengono i cosidetti «modi di velocità"; così sono chiamati dal Baños quei modi di propagazione per i quali la velocità $v$ è perpendicolare al piano formato dalla direzione del campo magnetico esterno e dal vettore di

(13) In realtá i due coefficienti sono legati dalla relazione $\beta T_{0}=\pi$ per cui l'annullarsi dell'uno porta all' annullarsi dell'altro. Consideriamo infatti le due relazioni di Thomson per corpi anisotropi nella forma (1.3) e (1.4) di [14] con $f(T) \equiv g(T) \equiv 0$; dopo sempliei passaggi si ricava $\varepsilon_{i k}=\frac{\pi_{i k}}{T}$, che nel caso isotropo ed omogeneo diventa $\varepsilon=\frac{\pi}{T}$. Quest'ultima equivale, nel nostro caso linearizzato, alla $\beta T_{0}=\pi$ (si veda [11], pag. 378).

(14) Ricordiamo che ̀े $\frac{\partial}{\partial z}=i k \cos \vartheta$ e quindi $\nabla t^{2}=-k^{2} \sin ^{2} \vartheta$. 
propagazione $\boldsymbol{k}$; inoltre tale velocità $v_{1}$ è caratterizzata dall'avere, per la (63)

$$
v_{s}=0 \quad \text { e } \nabla \cdot \boldsymbol{v}=0,
$$

ossia in tal caso il mezzo non esercita la sua comprimibilita. Introducendo questi valori nelle equazioni $(71),(72)$ e (73) si vede che, affinchè esse siano soddisfatte, occorre supporre $\Theta=$ cost.; la (74) invece fornisce

$$
\left(k_{L}^{2}-k^{2}\right)\left[k_{d}^{2}-i \alpha\left(k_{d}^{2}-k^{2}\right)\right]+k_{a}^{2}\left(k_{d}^{2}-k^{2}\right) k_{d}^{2}(1-i b)^{-1} k^{2} \sin ^{2} \theta=0 .
$$

Questa equazione coincide con la (13) di [7]; da essa si deducono due modi magnetoelastici distinti: noi li chiameremo modi di velocità isotermi, sottolineando il fatto che non sembrano possibili modi di velocita con $\theta$ variabile.

Sono invece possibili modi termici se si sceglie per la velocità la com. binazione

$$
\boldsymbol{v}=\boldsymbol{v}_{3} \cos \varphi-\boldsymbol{v}_{2} \sin \varphi
$$

dove $\varphi$, se reale, indica l'angolo che $v$ forma con il vettore $\boldsymbol{k}$ di propaga. zione e $v_{2}, v_{3}$ sono date da $\left(63_{2,3}\right)$; nella $(78)$ è messa in evidenza la parte solenoidale e quella irrotazionale della velocità. Questa espressione della velooità può anche scriversi

$$
\boldsymbol{v}=\left[\cos \varphi n+\left(\boldsymbol{i}_{2} \times \boldsymbol{n}\right) \sin \varphi\right] v_{0} \psi,
$$

da cai appaiono le seguenti proprietà:

$$
\left\{\begin{array}{l}
\boldsymbol{k} \cdot \boldsymbol{v}=k v_{0} \psi \cos \varphi \\
v_{z}=v_{0} \psi \cos (\theta+\varphi) \\
\boldsymbol{k} \times \boldsymbol{v} \cdot \boldsymbol{i}_{\mathrm{s}}=0
\end{array}\right.
$$

L' ultima delle (79) ci assicura subito che la (74) è senz'altro soddisfatta; dalla (72) si determina il parametro $\varphi$ in termini di $k$ : infatti sostituendo $\Theta$ in funzione di $\nabla \cdot v$ per mezzo della (71), da (72) si ottiene ${ }^{15}$ )

$$
\operatorname{tang} \theta \operatorname{tang} \varphi=\frac{k_{d}^{2}\left(k_{c}^{2}-k^{2}\right)}{k_{e}^{2}\left(k_{d}^{2}-k^{2}\right)}-i \frac{K^{2} \alpha^{2} T_{0} k_{d}^{2} k^{2}}{\rho \omega\left(i \omega \rho C_{\varepsilon}-x k^{2}\right)\left(k_{d}^{2}-k^{2}\right)} .
$$

Sostituendo invece $\nabla \cdot v$ in termini di $\theta$, sempre mediante la (71), nella

(15) La (89), in assenza di accoppiamento termoelastico $(x=0)$, coincide con la (15) di [7]. 
(79), si ricava

$$
\begin{gathered}
\left(i \omega \rho C_{\varepsilon}-x k^{2}\right)\left[\left(k^{2}-k_{L}^{2}\right)\left\{\left(k_{d}^{2}-k_{c}^{2}\right) k^{2} \cos ^{2} \theta+\left(k^{2}-k_{d}^{2}\right)\left[k_{c}^{2}-i a\left(k_{a}^{2}-k^{2}\right)\right]\right\}+\right. \\
\left.+k_{a}^{2}\left(k_{d}^{2}-k^{2}\right)\left(k_{o}^{2}-k^{2}\right)\right]+i \frac{K^{2} \alpha^{2} T_{0}}{\rho \omega} k_{c}^{2} k^{2}\left\{\left(k^{2}-k_{L}^{2}\right)\left[k_{d}^{2}-i a\left(k_{d}^{2}-k^{2}\right)\right]+\right. \\
\left.+k_{a}^{2}\left(k^{2}-k_{d}^{2}\right)+k_{d}^{2}\left(k_{L}^{2}-k^{2}\right) \sin ^{2} \theta\right\}=0 .
\end{gathered}
$$

La (81) è un'equazione di quarto grado in $k^{2}$ e quindi mette in evidenza la possibilità di quattro modi distinti magneto-termo-elastici. Osserviamo che in assenza di interazione termoelastica, $\alpha=0$, si presentano, ovviamente, un modo di natura puramente termica caratterizzato dal numero d'onda

$$
k^{2}=\frac{i \omega \rho C_{\varepsilon}}{\chi}
$$

e i tre modi magnetoelastici già studiati dal BAÑos [7]. Considerando piccolo l'apporto del termine in $\alpha^{2}$ si può pensare che i quattro modi di propagazione messi in luce dalla (81) non sono altro ehe $i$ tre modi magnetoelastici del Baños e quello termico relativo alla (82) modificati dalle proprietà ter. moelastiche del mezzo.

Studiamo ora la (81) in alcuni casi particolari.

Limite per $B_{0} \rightarrow 0$.

Se facciamo tendere a zero il campo magnetico esterno, l' equazione (81) si semplifica in quanto $\frac{1}{a} \rightarrow 0$ e $\frac{k_{a}^{2}}{a} \rightarrow \mu \omega \gamma$, e diventa

$$
\left(k_{d}^{2}-k^{2}\right)\left[\mu \omega \gamma-i\left(k_{L}^{2}-k^{2}\right)\right]\left\{\left(k_{c}^{2}-k^{2}\right)\left(i \omega \rho O_{\varepsilon}-x k^{2}\right)-i \frac{K^{2} \alpha^{2} T_{0}}{\rho \omega} k_{c}^{2} k^{2}\right\}=0 .
$$

Da questa si ottengono subito onde di distorsione, $k^{2}=k_{d}^{2}$, e onde di natura elettromagnetica in corrispondenza al numero d'onda

$$
k^{2}=k_{L}^{2}+i \mu \omega \gamma
$$

più precisamente in quest' ultimo caso si ha una composizione di onde elettromagnetiche con la velocita della luce e di onde elettromagnetiche superficiali (skin waves). La (83) dà inoltre

$$
\left(k_{c}^{2}-k^{2}\right)\left(i \omega \rho C_{\varepsilon}-\chi k^{2}\right)-i \frac{K^{2} \alpha^{2} T_{0}}{\rho \omega} k_{c}^{2} k^{2}=0 ;
$$

questa equazione mette in evidenza la possibilità di due modi distinti che, 
nel caso particolare di $\alpha=0$, si riducono ad onde di compressione $\theta$ ad onde termiche. Anzichè risolvere la (85), ci sembra più interessante studiarla nel caso in cui sia piccolo il coefficiente di conducibilità termica: $x \ll 1$. Riscriviamo allora la (85) nella forma

$$
\left(\omega \rho C_{\varepsilon}+\frac{K^{2} \alpha^{2} T_{0}}{\rho \omega} k_{c}^{2}\right) k^{2}-\omega \rho C_{\varepsilon} k_{c}^{2}=-i x\left(k^{2}-k_{c}^{2}\right) k^{2} .
$$

Indichiamo con

$$
k_{0}^{2}=\frac{k_{c}^{2}}{1+\frac{K^{2} \alpha^{2} T_{0}}{\rho^{2} \omega^{2} C_{\varepsilon}} k_{c}^{2}}
$$

il valore di $k^{2}$ che da $\left(8^{\prime}\right)$ si ottiene nel caso $x=0$ e che corrisponde a una onda di compressione modificata dalla interazione termoelastica; considerando poi il secondo membro della stessa equazione come una piccola perturba. zione, se ne ricava per una radice l'espressione

$$
k^{2} \approx k_{0}^{2}\left\{1+i x \rho \omega \frac{K^{2} \alpha^{2} T_{0} k_{c}^{4}}{\left(\rho^{2} \omega^{2} C_{\varepsilon}+K^{2} \alpha^{2} T_{0} k_{c}^{2}\right)^{2}}\right\}
$$

che rappresenta un'onda del tipo (86) lievemente attenuata dall'interazione termoelastica; per l'altra radice $k_{2}^{2}$, essendo ovviamente $k_{1}^{2} k_{2}^{2}=i \frac{\omega \rho C_{\varepsilon} k_{c}^{2}}{x}$, si ottiene $\left.{ }^{(16}\right)$ l'espressione approssimata

$$
k_{2}^{2} \approx i \frac{\omega \rho O_{\varepsilon}}{x}\left\{1+\frac{K^{2} \alpha^{2} T_{0} k_{o}^{2}}{\rho^{2} \omega^{2} C_{\varepsilon}}\right\}\left\{1-i x \rho \omega \frac{K^{2} \alpha^{2} T_{0} k_{c}^{4}}{\left(\rho^{2} \omega^{2} C_{\varepsilon}+K^{2} \alpha^{2} T_{0} k_{c}^{2}\right)^{2}}\right\}
$$

che è dominata dal fattore $i \frac{\omega_{\rho} C_{\varepsilon}}{x}$ e quindi corrisponde praticamente a una onda termica. Concludendo, quando il campo magnetico esterno tende a scom. parire $\left(B_{0} \longrightarrow 0\right)$ sono possibili quattro modi di propagazione per onde piane: due, quello di distorsione e quello etettromagnetico, non sono influenzati dall'interazione magneto-termo-elastica mentre gli altri due risentono delle proprietà termiche del mezzo; trascurando poi completamente gli effetti termici, $\alpha=0$ e $x=0$, questi due ultimi modi si ridacono a uno solo corrispondente ad onde elastiche di compressione.

\footnotetext{
${ }^{\left({ }^{6}\right)}$ Ricordiamo che per $x \ll 1$ si puo scrivere $\left\{1+i x p \omega \frac{K^{2} \alpha^{2} T_{0} k_{c}^{4}}{\left(\rho^{2} \omega^{2} C_{\varepsilon}+K^{2} x_{0} T_{0} k_{c}^{2}\right)^{2}}\right\}^{-1} \approx$ $\approx\left\{1-i x \rho \omega \frac{K^{2} \alpha^{2} T_{0} F^{4} c}{\left(\rho^{2}\left(\omega^{2} C_{\varepsilon}+K^{2} \alpha^{2} T_{0} k_{\varepsilon}^{2}\right)^{2}\right.}\right\}$.
} 
Si può poi anche vedere il comportamento dell'equazione (85) alle alte e basse frequenze. Alle basse frequenze, $\omega \ll 1$, si ottiene

$$
k^{2} \approx k_{e}^{2}\left\{1+i \frac{K^{2} \alpha^{2} T_{0}}{\rho \omega x}\right\}
$$

cioè un modo di compressione fortemente modificato da fattori termoelastici. Alle alte frequenze, $\omega \gg 1$, da (85) si ottiene invece

$$
k^{2}=k_{0}^{2} \quad \ominus \quad k^{2}=\frac{i \omega \rho C_{\varepsilon}}{x},
$$

ossia onde di compressione e onde termiche: in sostanza alle alte frequenze il corpo si comporta come in assenza di accoppiamento termoelastico.

Limite per $B_{0} \rightarrow \infty$.

Se si fa tendere $B_{0} \rightarrow \infty$ segue immediatamente che $k_{a}^{2} \rightarrow 0$ e $a \rightarrow 0$ e quindi la (81) assume l'espressione

$$
\left(k^{2}-k_{L}^{2}\right)\left\{\left(i \omega \rho C_{\varepsilon}-x k^{2}\right)\left[\left(k_{d}^{2}-k_{c}^{2}\right) k^{2} \cos ^{2} \theta+k_{d}^{2}\left(k^{2}-k_{d}^{2}\right)\right]+i \frac{K^{2} \alpha^{2} T_{0}}{\rho \omega} k_{c}^{2} k_{d}^{2} k^{2} \cos ^{2} \theta\right\}=0 .
$$

Da questa si ricava $k^{2}=k_{L}^{2}$, cioè un modo di natura elettromagnetica con una velocità uguale a quella della luce, (risultato questo abbastanza ovvio in quanto, facendo $B_{0} \rightarrow \infty$, si esaltano gli effetti elettromagnetici) e

$$
\left(i \omega \rho O_{\varepsilon}-x k^{2}\right)\left[\left(k_{d}^{2}-k_{c}^{2}\right) k^{2} \cos ^{2} \theta+k_{c}^{2}\left(k^{2}-k_{d}^{2}\right)\right]+i \frac{K^{2} \alpha^{2} T_{0}}{\rho \omega} k_{c}^{2} k_{d}^{2} k^{2} \cos ^{2} \theta=0 .
$$

Ora se in questa si trascura l'interazione termoelastica, facendo $\alpha=0$, si ottiene il modo termico relativo al numero d'onda (82) e un altro modo con numero d'onda dato da

$$
k^{2}=\frac{k_{c}^{2} k_{d}^{2}}{k_{d}^{2} \cos ^{2} \theta+k_{o}^{2} \sin ^{2} \theta}
$$

in quest'ultimo caso si tratta di una composizione di onde di compressione e di distorsione modificate anisotropicamente dalla forte interazione magnetoelastica e con una velocità di fase data da

$$
v_{\varphi}^{2}=V_{c}^{2} \cos ^{2} \theta+V_{d}^{2} \sin ^{2} \theta\left({ }^{17}\right) .
$$

Quindi da (92) si possono dedurre, almeno finchè il coefficiente $\alpha$ è abbastanza

(17) Per queste onde si veda [7]. 
piccolo, onde termiche ed elastiche alterate, rispetto ai numeri d'onda (82) e (93), in maniera anisotropa dalle proprietà termoelastiche del mezzo $(\alpha \neq 0)$.

Alle alte frequenze, $(\omega \gg 1)$, infine, sempre da (92) si ottengono ancora onde termiche ed elastiche come se si fosse in assenza di accoppiamento termoelastico $(\alpha=0)$, mentre alle basse frequenze $(\omega \ll 1)$ si ha

$$
k^{2} \approx \frac{k_{\theta}^{2} k_{d}^{2}}{k_{d}^{2} \cos ^{2} \theta+k_{c}^{2} \sin ^{2} \theta}\left[1+i \frac{K^{2} \alpha^{2} T_{0}}{\rho \omega x} \cos ^{2} \theta\right]
$$

cioè ancora onde elastiche che, rispetto al numero d'onda (93), sono forte. mente alterate dal fattore termico $\left[1+i \frac{K^{2} \alpha^{2} T_{0}}{\rho \omega x} \cos ^{2} \vartheta\right]\left({ }^{18}\right)$. Facciamo infine notare che questo fattore termico che altera le onde elastiche alle basse frequenze differisce solo per $\cos ^{2} \vartheta$ da quello che compare nella (86), relativo alle basse frequenze nel caso $B_{0} \longrightarrow 0$; questa differenza corrisponde fisicamente ad un comportamento anisotropo del mezzo causato dalla forte interazione magne. toelastica $\left(B_{0} \rightarrow \infty\right)$.

\section{8. - II caso: conducibilità elettrica infinita $(\gamma=\infty)$.}

Nel caso in cui il corpo in esame sia un perfetto conduttore dell' elettricità $(\gamma=\infty)$ invece della (5) si ha l'equazione

$$
\boldsymbol{E}=-\boldsymbol{v} \times \boldsymbol{B}+\beta \nabla T \text {. }
$$

Si può quindi, in questo caso, trasformare ulteriormente il termine in $\nabla \cdot \boldsymbol{j}$ che compare nell' equazione del calore (45') tenendo presente che è, per la (1),

$$
\nabla \cdot \boldsymbol{j}=-\varepsilon \frac{\partial}{\partial t}(\nabla \cdot E)
$$

e, per la $\left(5^{\prime}\right)$,

$$
\nabla \cdot \boldsymbol{E}=-\nabla \cdot(\boldsymbol{v} \times \boldsymbol{B})+\beta \nabla T=-\nabla \times \boldsymbol{v} \cdot \boldsymbol{B}+\nabla \times \boldsymbol{B} \cdot \boldsymbol{v}+\beta \nabla^{2} T .
$$

Prendendo di questa equazione la forma linearizzata e sostituendola in (96) si ottiene

$$
\nabla \cdot j=\varepsilon\left(\frac{\partial}{\partial t} \nabla \times v\right) \cdot B_{0}-\varepsilon \beta \nabla^{2} \frac{\partial \Theta}{\partial t}
$$

(18) Facciamo notare che per $\theta \simeq \frac{\pi}{2}$ si ha $k^{2}=k^{2}$. 
Di conseguenza l'equazione del ealore (45'), nel caso delle onde piane sinusoidali, fornisce

$$
\left[k^{2}\left(\chi-i \omega \varepsilon \beta^{2} T_{0}\right)-i \omega \rho O_{i}\right] \Theta=-K \alpha T_{0} \nabla \cdot v+i \omega \varepsilon \beta T_{0} B_{0}\left(\nabla \times v \cdot i_{3}\right) .
$$

Per quel che riguarda le altre equazioni si ha: la (68) rimane inalterata

$$
k_{e}^{2}\left(k_{d}^{2}-k^{2}\right) v_{z}=-\left(k_{d}^{2}-k_{e}^{2}\right) \frac{\partial}{\partial z}(\nabla \cdot v)-i \frac{K \alpha}{\rho \omega} k_{e}^{2} k_{d}^{2} \frac{\partial \Theta}{\partial z}
$$

la $(69)$ e la (70) diventano rispettivamente $\left({ }^{19}\right)$

$$
\begin{gathered}
\left\{\left(k_{L}^{2}-k^{2}\right)\left[\left(k_{d}^{2}-k_{c}^{2}\right) \frac{\partial^{2}}{\partial z^{2}}+k_{c}^{2}\left(k_{d}^{2}-k^{2}\right)\right]+k_{a}^{2}\left(k_{d}^{2}-k^{2}\right)\left(k_{c}^{2}-k^{2}\right)\right\}(\nabla \cdot \boldsymbol{v})= \\
=-i \frac{K \alpha}{\rho \omega} k_{c}^{2}\left[\left(k_{L}^{2}-k^{2}\right) k_{d}^{2} \frac{\partial^{2}}{\partial z^{2}}-k_{a}^{2}\left(k_{d}^{2}-k^{2}\right) k^{2}\right] \Theta \\
{\left[k_{d}^{2} \frac{\partial^{2}}{\partial z^{2}}+k_{L}^{2} k_{d}^{2}+k_{a}^{2}\left(k_{d}^{2}-k^{2}\right)\right]\left(\nabla \times \boldsymbol{v} \cdot \boldsymbol{i}_{3}\right)=\frac{\beta}{B_{0}} k_{d}^{2} k_{L}^{2} \nabla_{t}^{2} \Theta .}
\end{gathered}
$$

Studiamo innanzitatto i modi di velocità, $v=v_{1}$.

Ricordando le (76) si vede subito che la (68) può essere soddisfatta in due casi : $\left.\left.1^{\circ}\right) \alpha=0,2^{\circ}\right) \frac{\partial \theta}{\partial z}=0$.

10) Se si pone $\alpha=0$ la $(100)$ è automaticamente verificata mentre la (99) diventa

$$
\left[k^{2}\left(x-i \omega \varepsilon \beta^{2} T_{0}\right)-i \omega \rho C_{\varepsilon}\right] \Theta=i \omega \varepsilon \beta T_{0} B_{0}\left(\nabla \times v \cdot i_{3}\right) .
$$

Eliminando $\Theta$ fra la (102) e la (101) si ottiene un' equazione dalla quale, an. nullando il coefficiente di $\nabla \times v \cdot i_{3}$, si ricava

$$
\begin{gathered}
{\left[k_{a}^{2}\left(k_{d}^{2}-k^{2}\right)-k_{d}^{2} k^{2} \cos ^{2} \theta\right]\left[k^{2}\left(x-i \omega \varepsilon \beta^{2} T_{0}\right)-i \omega \rho C_{\varepsilon}\right]+} \\
+k_{L}^{2} k_{d}^{2}\left[k^{2}\left(x-i \omega \varepsilon \beta^{2} T_{0} \cos ^{2} \theta\right)-i \omega \rho C_{\varepsilon}\right]=0 .
\end{gathered}
$$

Essendo questa di secondo grado in $k^{2}$, si hanno dunque due modi distinti di velocità che, a differenza di quanto accade nel caso già visto di $\beta=0$ e $\gamma \neq \infty$, sono anche modi termici, cioè non si richiede necessariamente che sia $\Theta=$ cost. Si può poi notare che, nel caso $\gamma=\infty$, l'interazione termoelettrica $(\beta \neq 0)$ è associata alla corrente di spostamento $(\varepsilon \neq 0)$; se trascuriamo anche la corrente di spostamento $\left(\varepsilon=0, k_{L}^{2}=0\right)$, da (103) si ricava un modo

(19) Ricordiamo che nel ease $\gamma=\infty$ si ha $a=b=0$. 
puramente termico con numero d'onda $k^{2}=\frac{i \omega \rho C_{s}}{x}$ e un modo magnetoelastico, anisotropo per effetto dell'interazione magnetoelastica, con numero d'onda

$$
k^{2}=\frac{k_{d}^{2} k_{d}^{2}}{k_{a}^{2}+k_{d}^{2} \cos ^{2} \vartheta} .
$$

Ancora da (103), se consideriamo onde piane propagantesi nella direzione del campo magnetico esterno, $\vartheta=0$, in modo da avere an comportamento isotropo del mezzo, si ottiene

$$
\left[k_{a}^{2}\left(k_{t}^{2}-k^{2}\right)-k_{d}^{2} k^{2}+k_{L}^{2} k_{d}^{2}\right]\left[k^{2}\left(x-i \omega \varepsilon \beta^{2} T_{0}\right)-i \omega \rho C_{\varepsilon}\right]=0
$$

da cui si deducono onde termoelettriche, in corrispondenza al valore

$$
k^{2}=\frac{i \omega \rho C_{\varepsilon}}{x-i \omega \varepsilon \beta^{2} T_{0}}
$$

e onde con numero d'onda dato da

$$
k^{2}=\frac{k_{d}^{2}\left(k_{a}^{2}+k_{L}^{2}\right)}{k_{a}^{2}+k_{d}^{2}}
$$

di natura elastica ed elettromagnetica.

$2^{\circ}$ ) Se si ammette invece che sia $\frac{\partial \Theta}{\partial z}=0$ (e quindi, per tutte le grandezze, $\frac{\partial}{\partial z}=i k \cos \theta=0$ il che equivale a supporre $\cos \vartheta=0$ ), che è l'altro caso in cui è soddisfatta la (68), per modi di velocità $v=v_{1}$, da (100) segue

$$
i \frac{K \alpha}{\rho \omega} k_{c}^{2} k_{a l}^{2}\left(k_{d}^{2}-k^{2}\right) k^{2}=0
$$

mentre la (101) diventa

$$
\left[k_{L}^{2} k_{d}^{2}+k_{a}^{2}\left(k_{d}^{2}-k^{2}\right)\right]\left(\nabla \times v \cdot i_{3}\right)=-\frac{\beta}{B_{0}} k_{d}^{2} k_{L}^{2} k^{2} \theta
$$

infine la (99) ha ancora la forma (102). Eliminando $\Theta$ fra la (109) e la (99) si ottiene

$$
k_{a}^{2}\left(k_{d}^{2}-k^{2}\right)\left[k^{2}\left(x-i \omega \varepsilon \beta^{2} T_{0}\right)-i \omega \rho O_{\varepsilon}\right]+k_{L}^{2} k_{d}^{2}\left[k^{2} x-i \omega \rho C_{\mathrm{\varepsilon}}\right]=0 .
$$

Si tratta quindi di risolvere il sistema formato da (108) e (110); si vede subito che questo sistema ammette la soluzione $k^{2}=k_{d}^{2}$ nel caso in cui si trascuri 
la corrente di spostamento $(\varepsilon=0)$; oppure, facendo $B_{0} \rightarrow \infty$ (da cui segue $\left.k_{a}^{2} \rightarrow 0\right)$ la $(108)$ è verificata per qualunque $k^{2}$ mentre la $(110)$ dà il valore $k^{2}=\frac{i \omega \rho C_{\varepsilon}}{x}$, corrispondente a un modo termico. Concludendo, nel caso $\frac{\partial \theta}{\partial z}=0$, che fisicamente corrisponde ad onde piane propagantesi in direzione normale al campo magnetico esterno, si possono avere i seguenti modi di velocità: onde di distorsione nel caso in cui si trascuri la corrente di spostamento, $\varepsilon=0$, onde termiche nel caso di un forte campo magnetico primario, $B_{0} \rightarrow \infty$.

Per quel che riguarda invece i modi di velocità isotermi, $\Theta=$ cost., si ha: le equazioni (68) e (100) sono verificate, la (101) e la (99) diventano rispettivamente

$$
\begin{gathered}
-k_{d}^{2} k^{2} \cos ^{2} \theta+k_{L}^{2} k_{d}^{2}+k_{a}^{2}\left(k_{d}^{2}-k^{2}\right)=0, \\
i \omega \varepsilon \beta T_{0} B_{0}=0 .
\end{gathered}
$$

Questo sistema ammette quindi le seguenti soluzioni: $k^{2}=\frac{k_{a}^{2} k_{d}^{2}}{k_{a}^{2}+k_{d}^{2} \cos ^{2} \theta}$ nel caso di $\varepsilon=0, k=\frac{k_{d}^{2}\left(k_{a}^{2}+k_{L}^{2}\right)}{k_{a}^{2}+k_{d}^{2} \cos ^{2} \theta}$ nel caso di $\beta=0$. Quindi si può dire che sono possibili i seguenti modi di velocità isotermi: un modo magnetoelastico anisotropo se si trascura la currente elettrica di spostamento $(\varepsilon=0)$, un modo pure anisotropo di natura elastica ed elettromagnetica se si trascura l'interazione termoelettrica $(\beta=0)$. Senza le ipotesi $\varepsilon=0$ o $\beta=0$ non sono invece possibili modi di velocità isotermi.

Scegliamo ora per la velocità la combinazione (78), cioè $v=\boldsymbol{v}_{\mathbf{2}} \cos \varphi-$ - $\boldsymbol{v}_{2} \sin \varphi$, che gode delle proprietà (79); ricordiamo che il sistema da stu. diare è costituito ancora dalle equazioni (99), (68), (100) e (101). Dalla (101), per la $(79)$, si ha subito

$$
\left(\frac{\beta}{B_{0}} k_{d}^{2} k_{L}^{2} k^{2} \sin ^{2} \vartheta \theta=0 .\right.
$$

Si possono quindi avere diversi casi particolari: il caso $\beta=0$ è già stato studiato nel n. 7 (basta aggiungere l'ipotesi $\gamma=\infty$ ); il caso $\varepsilon=0$ porta alla equazione (80) tale e quale e alla (81) in cui faccia $k_{L}^{2}=a=0$ (si hanno quindi tre modi distinti anzichè quattro come segue dalla (81)); anche considerando il limite $B_{0} \rightarrow \infty$ si ottengono di nuovo la (80) inalterata e la (81) con $k_{a}^{2}=a=0$. Si ottiene invece qualcosa di nuovo considerando onde ohe si propagano nella direzione del campo magnetico esterno, ciò̀ per $\mathscr{\vartheta}=0$. Eliminando $\Theta$ tra la (99) e la (68) e tra la (99) e la (100) si ricava ri- 
spettivamente

$$
\begin{gathered}
\left(k_{c}^{2}-k^{2}\right)\left[k^{2}\left(x-i \omega \varepsilon \beta^{2} T_{0}\right)-i \omega \rho C_{\varepsilon}\right]+i \frac{K^{2} \alpha^{2} T_{0}}{\rho \omega} k_{c}^{2} k^{2}=0 \\
{\left[\left(k_{L}^{2}-k^{2}\right) k_{d}^{2}+k_{a}^{2}\left(k_{d}^{2}-k^{2}\right)\right]\left\{\left(k_{c}^{2}-k^{2}\right)\left[k^{2}\left(x-i \omega \varepsilon \beta^{2} T_{0}\right)-i \omega \rho C_{\varepsilon}\right]+\right.} \\
\left.+i \frac{K^{2} \alpha^{2} T_{0}}{\rho \omega} k_{c}^{2} k^{2}\right\}=0
\end{gathered}
$$

Questo sistema, ovviamente, ha per soluzione le soluzioni della (114): si tratta di due modi di propagazione, uno relativo ad onde di compressione e l'altro ad onde di natura termoelettrica con numeri d'onda alterati, rispetto a $k_{c}^{2} \theta$ a quello dato dalla (106), a seguito delle proprietà termoelastiche del mezzo $(\alpha \neq 0)$. Notiamo infine che la (114) contiene come caso particolare per $\varepsilon=0$ o $\beta=0$, la $(85)$, che è relativa al limite $B_{0} \rightarrow 0$ nel caso $\beta=0$ : c'è quindi una certa analogia tra quel caso e quest'nltimo corrispondente a propagazione per onde piane nella direzione del campo magnetico esterno in un mezzo perfetto conduttore dell' elettricità.

\section{BIBLIOGRAFIA}

[1] G. Paria, On magneto.thermo-elastic plane waves, Proc. Camb. Phil. Soc. 58 (1962), 527.521 .

[2] A.J. WLLLSON, The propagation of magneto-thermo-elastic plane waves, Proe. Camb. Phil. Soc, 59 (1963), 483.488.

[3] C. M. Purushomama, Magnetorthermo elastic plane waves, Proe. Camb. Phil. Soe. 61 (1965), 939.944.

[4] A. BÃ̃̃os, Jr., Fundamental Wave Functions in an unbounded magneto.hydrodynamic field. I General theory, Phys. Rev., 97 (195̃5), 1455.43.

[5] - - Magneto.hydrodynamic waves in incompressible and compressible fluids, Proc. Roy. Soc., A, 283, (1955), 350-67.

[6] - -, Magneto-hydrodynamic waves in compressible fluid with finite viscosity and heat conductivity, Int. Astr. Un. Symp., n. 6 (1958), 15.26.

[7] - - Normal modes characteriaing magneto-elastic plane waves, Phys. Rev. 104 (1956), 300-305.

[8] L. D. Landau, E. M. Lifshimz, Electrodynamics of continuons media, Pergamon Press, Oxford, (1960).

[9] W. Now Ackx, Thermoelasticity, Int. Ser. of Monografs on Aeronauties and Astronautics, Addison Wesley, (1962). 
[10] I. I. Gol'Denblat, Some problems of the mechanics of deformable media, P. Noordholf LTD., Groningen, (1962).

[11] S. KALISKI, W. NOWACKI, The reciprocity theorem of magneto-thermo-elasticity. II. Real Conductors, Bull. Acad. Polon. Sci., Ser. sci. techn,, 13, (1965), 377-84.

[12] - - - - Combined elastic and electromagnetic waves produced by thermal shock in the case of a medium of finite electric conductivity, Bull. Aoad. Polon. SCI., Sor. sci. techn., 10, (1962), 160.f8.

[13] S. KaLISki, Wave epuations of thermo-electromagnetoelasticity, Proc. Vibr. Probl. 3, 6. (1965), 231.65.

[14] J. MEIXNer, Thermodynamische und kinetische Behandlung der thermoelektrischen Ef: fekte im Magnetfeld, Ann. der Physic, 35, (1939), 701-34. 